\title{
Low Burst Promoting Activity Production by Phytohemagglutinin-Stimulated Mononuclear Cells of Cord Blood
}

\author{
YOSHITAKA IWAI, TAKESHI MIYANOMAE, MAYUMI TANAKA, TOMOHIDE YOSHIMURA, \\ MITSUFUMI MAYUMI, AND HARUKI MIKAWA \\ Department of Pediatrics, Kyoto University, Kyoto, Japan
}

\begin{abstract}
Burst-promoting activity (BPA) produced by phytohemagglutinin(PHA)-stimulated cord blood mononuclear cells (MNC) was examined using the two-stage cell culture assays. Burst-promoting activity was measured as the increase in the number of early erythroid progenitor cells in 2-day incubation of peripheral blood MNC with or without the conditioned medium of PHA-stimulated MNC (PHA-LCM). Burst-promoting activity in PHA-LCM of cord blood was significantly lower than that of adult blood $(37 \pm 13$ versus $105 \pm 19 \%$, mean $\pm \mathrm{SD}, p<0.01)$. No elevation of inhibitors to the erythroid colony growth was noted in PHA-LCM of cord blood. In contrary, the response of cord blood MNC to PHA was similar to that of adult blood MNC, as determined by colony-stimulating activity production and cell proliferation. These results showed that burst-promoting activity production by PHAstimulated MNC of cord blood was lower than that of adult blood. (Pediatr Res 21: 285-288, 1987)
\end{abstract}

\section{Abbreviations}

BPA, burst-promoting activity

PHA-LCM, phytohemagglutinin-stimulated mononuclear cells conditioned medium

CSA, colony-stimulating activity

MNC, mononuclear cells

FCS, fetal calf serum

BFU-E, erythroid burst-forming unit

CFU-GM, granulocyte, monocyte colony forming unit

IMDM, Iscoves' modified Dulbecco's medium

${ }^{3} \mathrm{H}-\mathrm{TdR}$, tritiated-thymidine

BPA is considered to be a humoral regulator of erythropoiesis different from that of erythropoietin (1). BPA was reported to regulate the early stage of erythropoiesis whereas erythropoietin regulates the late stage of erythropoiesis. BPA was obtained from many different sources including conditioned medium of $\mathrm{T}$ cells (2), monocytes (3), large granular lymphocytes (4), bone marrow cells (5), and mitogen stimulated cells $(6,7)$.

Erythropoiesis in the early infantile period shows marked changes. The high values of hemoglobin at birth decrease rapidly and reach a nadir at about 2 months of age. Thereafter, erythropoiesis is reactivated and the hemoglobin levels return to the normal level of children. Several studies found a relationship

Received June 23, 1986; accepted October 27, 1986

Reprint requests and correspondence to Yoshitaka Iwai, M.D., Department of Pediatrics, Kyoto University, Kawahara-cho 53, Shogoin Sakyo, Kyoto, Kyoto Prefecture 606, Japan. between the serum erythropoietin level and erythropoiesis in this period (8-10). However, the detailed mechanism of humoral regulation of erythropoiesis in this period is still unclear, and the ability of BPA production by MNC in the neonatal period has not been evaluated. In the present study, we investigated the BPA production by PHA-stimulated MNC of cord blood in comparison with that of adult peripheral blood and showed that BPA production by cord blood MNC were markedly lower than that of adult blood.

\section{MATERIALS AND METHODS}

Subjects. Cord blood was drawn from umbilical cords of normal neonates at 37-41 wk gestations with informed consent of their parents. Adult peripheral venous blood was obtained from healthy volunteers.

Preparation of conditioned medium. MNC were separated from the heparinized blood by Ficoll-Hypaque density gradient and washed twice with IMDM (GIBCO, Grand Island, NY). Unfractionated MNC were suspended at a concentration of $1 \times$ $10^{6}$ cells $/ \mathrm{ml}$ in IMDM supplemented with $20 \%$ FCS (Irvine Scientific, Santa Ana, CA) and 1\% PHA-M (Sigma, St. Louis, $\mathrm{MO})$. The cells were cultured in Falcon culture frascoves for 5 days at $37^{\circ} \mathrm{C}$ in a humidified atomosphere of $5 \% \mathrm{CO}_{2}$ in air. After 5 days of culture, the medium was aspirated from the culture frascoves, centrifuged at $400 \times g$ for $10 \mathrm{~min}$, passed through a $0.22 \mu \mathrm{m}$ millipore filter, and frozen at $-20^{\circ} \mathrm{C}$ until use for BPA and CSA assay.

$B P A$ assay. Dukes' two-stage assay (11) was used with a little modification. MNC were separated from adult peripheral blood and adherent cells were removed as previously described (12). Nonadherent cells were suspended at a concentration of $3 \times 10^{5}$ cells $/ \mathrm{ml}$ in $4 \mathrm{ml}$ IMDM containing $20 \% \mathrm{FCS}, 5 \times 10^{-5} \mathrm{M}$ 2-mercaptoethanol and several concentrations of PHA-LCM. As negative controls, the cells were incubated without the addition of PHA-LCM and were carried out in every experiment. After 2-day culture of the nonadherent MNC in $17 \times 100 \mathrm{~mm}$ Falcon capped tissue culture tubes, the cells were collected by centrifugation at $300 \times g$ for $10 \mathrm{~min}$ and resuspended in $4 \mathrm{ml}$ fresh medium containing $30 \%$ FCS, $5 \times 10^{-5} \mathrm{M}$ 2-mercaptoethanol, $1 \%$ bovine serum albumin (Sigma), $0.9 \%$ methylcellulose, and 1 $\mathrm{U} / \mathrm{ml}$ erythropoietin ( $57 \mathrm{U} / \mathrm{mg}$, Toyobo, Osaka, Japan). For the second stage of the assay, $1-\mathrm{ml}$ aliquots of these cell suspensions were incubated in $35 \times 100 \mathrm{~mm}$ Falcon tissue culture dishes for 10 12 days, and then evaluated for BFU-E colonies. Colonies consisting of three or more subcolonies of erythroid cells or large single accumulation of erythroid cells ( $>200$ cells) were defined as BFU-E-derived colonies. BPA response was defined as the increases in BFU-E colonies observed in cultures containing PHA-LCM during the suspension culture stage over the numbers of colonies found in negative controls. BPA response of each 
sample was expressed as a percentage of the activity to standard PHA-LCM samples in our laboratories. The interassay variance in the assays was less than $15 \%$.

CSA assay. Fresh bone marrow cells were obtained from hematologically normal patients who were undergoing routine evaluation. Nonadherent MNC were separated by the FicollHypaque procedure and plastic adherence. The nonadherent MNC were incubated at $1 \times 10^{5}$ cells $/ \mathrm{ml}$ in IMDM containing $20 \%$ FCS, $0.3 \%$ agar, and PHA-LCM at several concentrations in $35 \times 100 \mathrm{~mm}$ Falcon culture dishes for 9 days. CFU-GM colonies were evaluated by counting colonies containing 40 or more granulocytes-monocytes. CSA was expressed as the number of CFU-GM colonies per $1 \times 10^{5} \mathrm{MNC}$.

Assay for ${ }^{3} H-T d R$ uptake. MNC at a concentration of $1 \times 10^{6}$ cells/ml were incubated in IMDM containing $20 \%$ FCS and $1 \%$ PHA-M in Falcon 96-well flat bottomed microplates. After 48 $\mathrm{h}$, the cultures were pulsed for $6 \mathrm{~h}$ with $1 \mu \mathrm{Ci} /$ well ${ }^{3} \mathrm{H}-\mathrm{TdR}$ (New England Nuclear, Boston, MA) and harvested onto glass fiber filter strips. Incorporation of ${ }^{3} \mathrm{H}-\mathrm{TdR}$ was determined by liquid scintillation spectrometry.

Statistical analysis. In all assays, differences were evaluated by Students' $t$ test.

\section{RESULTS}

Dose response of BFU-E colony growth to PHA-LCM. Effects of PHA-LCM of adult and cord blood on BFU-E colony formation are shown in Figure 1. The numbers of BFU-E colonies increased at the PHA-LCM concentrations from 0 to 5\% and the maximum response was obtained at $5 \%$ in every sample. Therefore, this concentration was used for the following BPA assay.

Because PHA-M might be contaminated in the PHA-LCM and the cultures with 5\% PHA-LCM might contain at most $0.05 \%$ PHA-M, $0.05-0.1 \%$ PHA-M was added to the control suspension cultures in order to examine whether these low concentrations of PHA-M had any effects on the increase of the early erythroid progenitor cells. These concentrations of PHA$M$ did not show any significant effects on the increase of BFU-E colonies (data not shown). Therefore, negative controls were incubated without the addition of PHA-M in the following experiments.

BPA assay. BPA in the PHA-LCM of cord blood and adult blood is shown in Figure 2. The mean value of BPA in the PHALCM of cord blood was $37 \pm 13 \%$ (mean \pm SD), whereas that of BPA in the PHA-LCM of adult blood was $105 \pm 19 \%$. The difference was significant $(p<0.01)$. It is well known that many conditioned media might contain two opposite activities, enhancing and inhibiting. Because BPA in the PHA-LCM might reflect the net activity of positive BPA and possible inhibitors, it was possible that the low BPA in the PHA-LCM of cord blood was due to the presence of inhibitors in the PHA-LCM although the PHA-LCM of cord blood contained as much BPA as that in the PHA-LCM of adult blood. To rule out this possibility, we examined the inhibitory activity of the PHA-LCM of cord blood.

In the first experiments, 5\% PHA-LCM of cord blood was added together with adult PHA-LCM in the 2-day incubation to examine whether the amount of inhibitors in cord blood PHALCM was sufficient to influence the action of adult PHA-LCM. The cord blood PHA-LCM that showed low BPA by themselves, did not cause significant inhibition in BFU-E colony growth induced by adult PHA-LCM (Table 1).

In the second experiments, the inhibitory effects of cord blood PHA-LCM were examined in one-stage BFU-E colony assay. PHA-LCM of cord blood was used at a concentration of $10 \%$ because our preliminary experiments revealed that inhibitory effects of the conditioned medium was generally detected at concentrations higher than $5 \%$ in one-stage assay (data not shown). As shown in Figure 3, 10\% PHA-LCM of cord blood did not inhibit BFU-E colony growth in the one stage BFU-E
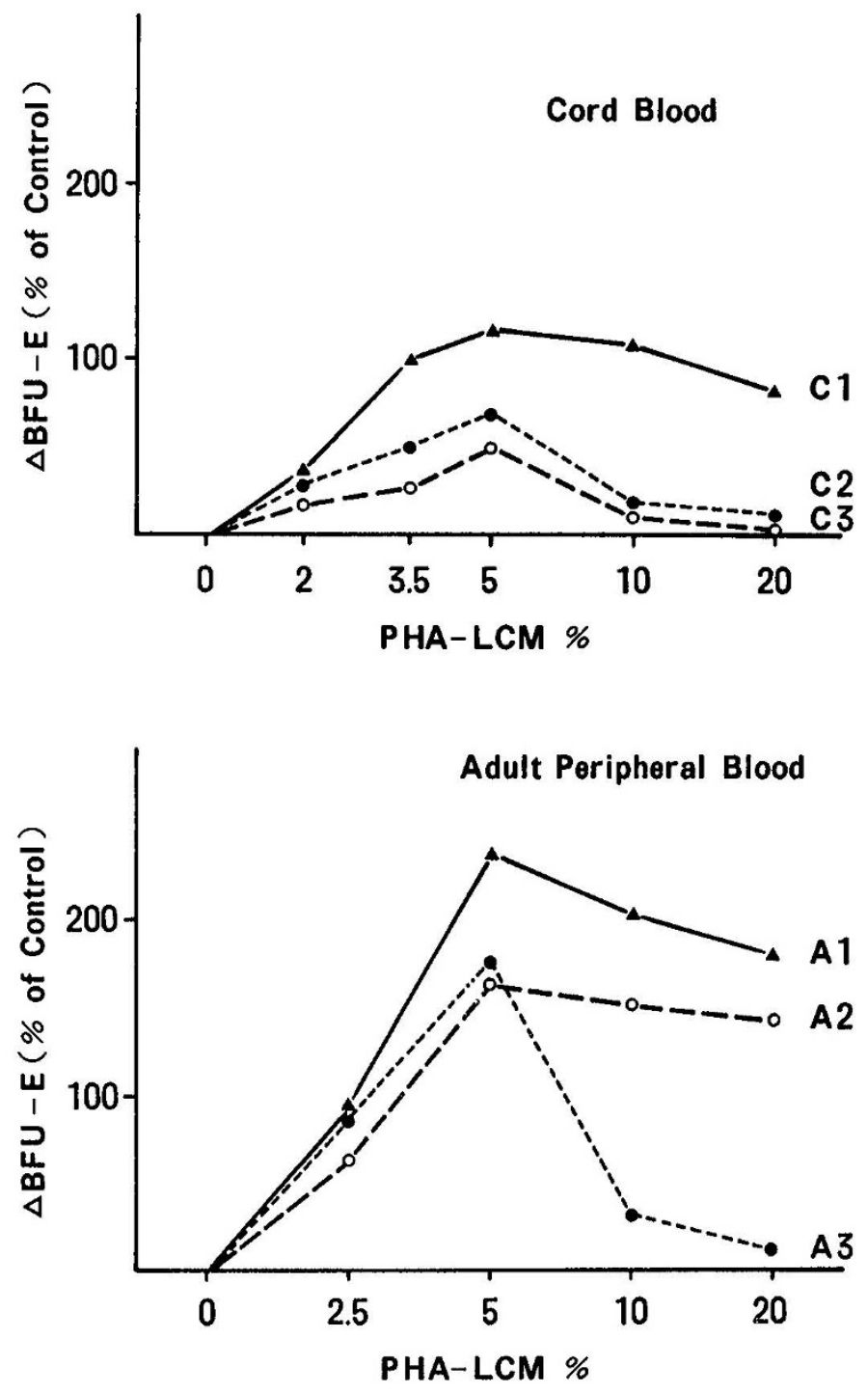

Fig. 1. Dose response of BFU-E colony growth to PHA-LCM of cord blood and adult blood. MNC were cultured with or without PHA-CLM for 2 days and then analyzed for BFU-E colony growth. $\triangle \mathrm{BFU}-\mathrm{E}$; (numbers of BFU-E in the cultures) - (numbers of the BFU-E in the control culture maintained without PHA-LCM). $\mathrm{Cl}, \mathrm{C} 2, \mathrm{C} 3$, cord blood; A1, A2, A3, adult blood.

assay, although neither 10\% PHA-LCM of adult blood nor $10 \%$ PHA-LCM of cord blood showed an additive BPA activity. It has been reported that exogenously added BPA causes only a slight increase in the numbers of BFU-E colonies in this assay system because crude erythropoietin and endogenously produced BPA from MNC showed submaximal BPA activities (11).

These results showed that the PHA-LCM prepared in this study did not contain an excess of inhibitory factors against BFU-E colony growth and suggested that the low BPA in the PHA-LCM of cord blood was not due to contaminating inhibitors.

CSA assay. The PHA-LCM of cord and adult blood was examined for the ability to stimulate CFU-GM colony growth (Table 2). Maximum CSA response was obtained with 5-10\% PHA-LCM and some samples showed inhibitory effect to CFUGM colony growth at concentrations of more than $10 \%$. Therefore, we used 5\% PHA-LCM for detecting CSA. CSA in PHALCM is shown in Table 1. There was no significant difference in CSA activities between the PHA-LCM of cord blood and the PHA-LCM of adult blood. 


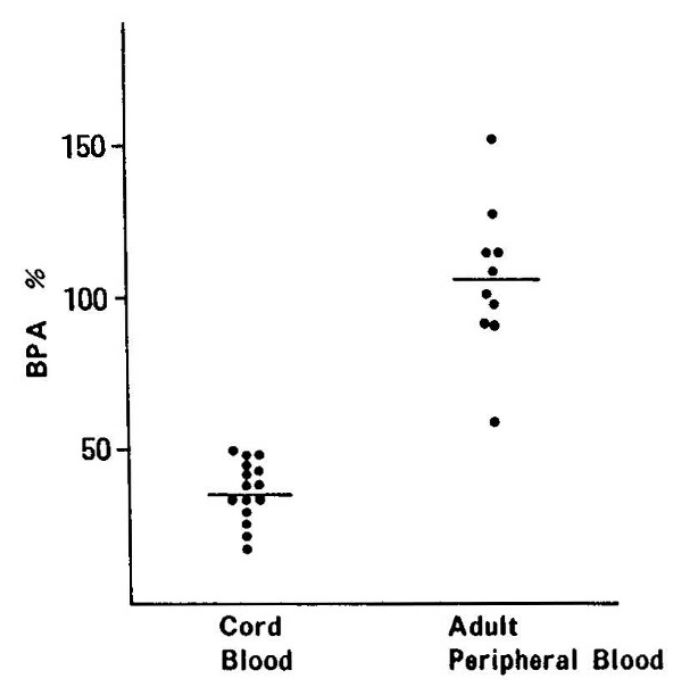

Fig. 2. BPA in PHA-LCM of cord blood and adult blood. BPA is expressed as a percentage of activity to standard PHA-LCM. Horizonial lines denote mean values.

Table 1. Lack of significant inhibitors in cord blood PHA-LCM detected by the two-stage BPA assay system*

\begin{tabular}{lcc}
\hline & \multicolumn{2}{c}{$\begin{array}{c}\text { BFU-E derived colonies } \\
\text { (per } 3 \times 10^{5} \text { cells) }\end{array}$} \\
\cline { 2 - 3 } Additions to suspension culture & Experiment 1 & Experiment 2 \\
\hline None (control) & 38 & 26 \\
$5 \%$ adult PHA-LCM & 122 & 93 \\
10\% adult PHA-LCM & 108 & 84 \\
$5 \%$ adult PHA-LCM + 5\% & & \\
$\quad$ other adult PHA-LCM & 100 & 82 \\
No. 1 & 117 & 85 \\
No. 2 & 102 & 78 \\
No. 3 & & \\
5\% adult PHA-LCM + 5\% & & \\
cord blood PHA-LCM & 106 & 81 \\
No. 1 & 101 & 81 \\
No. 2 & 96 & 75 \\
No. 3 & & \\
$5 \%$ cord blood PHA-LCM & ND & 54 \\
No. 1 & ND & 43 \\
No. 2 & ND & 39 \\
No. 3 &
\end{tabular}

* PHA-LCM of cord blood or adult blood was added to the first stage culture where peripheral blood MNC were cultured with or without PHA-LCM of adult blood for 2 days. BFU-E of the cultures were then evaluated. Each value shows the mean of three replicates. ND; not done.

${ }^{3} \mathrm{H}$-TdR uptake assay. Mean ${ }^{3} \mathrm{H}-\mathrm{TdR}$ uptakes of PHA-stimulated MNC of cord blood and adult blood were 168,000 $\pm 46,000$ cpm $(n=8)$ and $157,000 \pm 35,000 \mathrm{cpm}(n=7)$, respectively. There was no significant difference between these two groups.

\section{DISCUSSION}

In this study, we showed that BPA in PHA-LCM of cord blood MNC was significantly lower than that of adult blood MNC. The low BPA in PHA-LCM of cord blood seemed due to the decreased ability of cord blood MNC to produce BPA but not due to contaminating inhibitors. We used Dukes' two-stage assay (11) with a little modification for detecting BPA. This assay system seems better than previously reported one-stage assay (13-15) where crude erythropoietin with or without samples to be examined was added to the culture and the effect of the

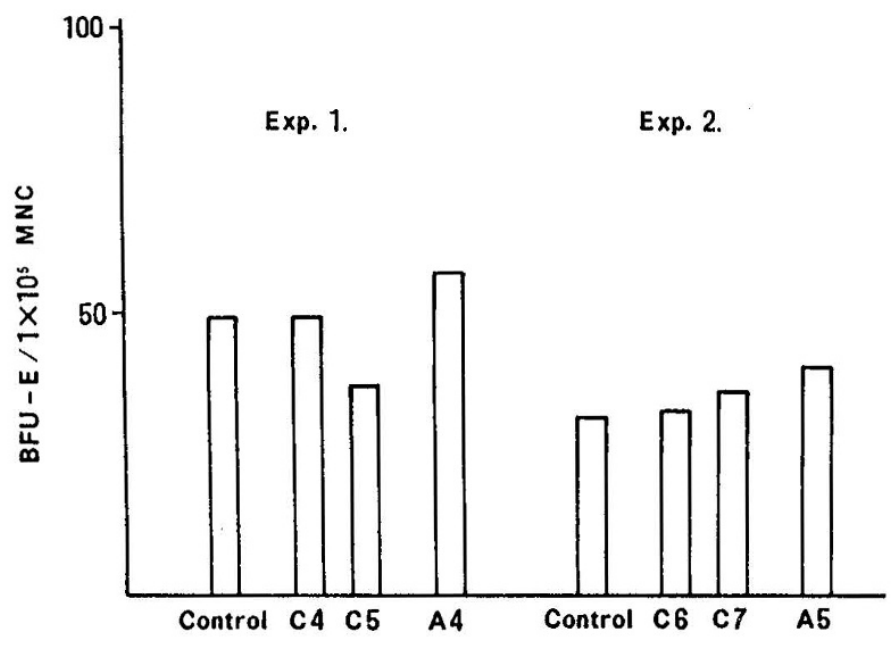

Fig. 3. Detection of inhibitors in PHA-LCM to erythroid colony growth. PHA-LCM was added at the final concentration of $10 \%$ to mononuclear cells in one-step BFU-E colony assay where $2 \times 10^{5}$ mononuclear cells were incubated in IMDM containing 30\% FCS, $1 \%$ bovine serum albumin, $5 \times 10^{-5} \mathrm{M} 2$-mercaptoethanol, $0.9 \%$ methylcellulose, and $1 \mathrm{U} / \mathrm{ml}$ erythropoietin for 10-12 days. Control, cultured without PHA-LCM; C4-C7, cord blood; A4, A5, adult blood.

Table 2. Colony-stimulating activity in PHA-LCM*

\begin{tabular}{llll}
\hline & \multicolumn{2}{c}{ CFU-GM per $1 \times 10^{5}$ MNC } & \\
\cline { 2 - 3 } & \multicolumn{1}{c}{ Cord blood } & \multicolumn{1}{c}{ Adult blood } & \\
\hline Experiment 1 & $68 \pm 32(n=6)$ & $67 \pm 34(n=5)$ & NS \\
Experiment 2 & $48 \pm 32(n=11)$ & $58 \pm 37(n=7)$ & NS \\
Experiment 3 & $71 \pm 29(n=15)$ & $60 \pm 32(n=10)$ & NS \\
\hline
\end{tabular}

$*$ Data were expressed as mean \pm SD. NS; not significant. Only a few colonies (0-3 per $\left.1 \times 10^{5} \mathrm{MNC}\right)$ were induced without PHA-LCM.

samples was compared with that of crude erythropoietin. However, because crude erythropoietin itself has significant BPA, none of these assays seems to be suitable to quantitate BPA. Our modified Dukes' two-stage assay system that did not use erythropoietin in the first stage culture, is thus better than these onestage assays for quantitation of BPA.

The mechanism of low BPA production in PHA-stimulated MNC of cord blood remains to be elucidated. A possible explanation is a low responsiveness of cord blood MNC to PHA stimulation. However, this explanation seems unlikely because there was no significant difference in ${ }^{3} \mathrm{H}-\mathrm{TdR}$ uptake or CSA production between PHA-stimulated MNC of cord blood and that of adult blood. Several studies found a suppressive effect of $\mathrm{T}$ cells and diminished function of monocytes of cord blood in other experimental systems $(16,17)$. These characteristics of cord blood MNC may have some role in low BPA production of cord blood. Further studies are now in progress to investigate the contribution of $\mathrm{T}$ cells and monocytes in the low BPA production by cord blood MNC.

It will be of interest to know whether low BPA production of cord blood $\mathrm{MNC}$ is responsible to the suppressed erythropoiesis in the neonatal period. Although BPA has been considered to regulate the early stage of erythropoiesis in vitro, the physiological role of BPA in vivo is still obscure. Further studies are necessary to clarify the relationship of the serum BPA level and BPA production of MNC to erythropoiesis in early infancy.

\section{REFERENCES}

1. Iscove NN, Ruitsh CA, William SN, Guilbert LJ 1982 Molecules stimulating early red cell, granulocyte, macrofage, and megakaryocyte precursors in culture; similarity in size, hydrophobicity and charge. J Cell Physiol [Suppl] 1:65-78 
288

IWAI $E T A L$.

2. Nathan DG, Chess L, Hellman DG, Clarke B, Beard J, Merles E, Housman BE 1978 Human erythroid burst forming unit: T-cell requirement for proliferation in vito. J Exp Med 147:324-339

3. Zucherman KS 1980 Stimulation of human BFU-E by products of human monocytes and lymphocytes. Exp Hematol 8:924-932

4. Pistoia BV, Ghio R, Nocera A, Leprini A, Perata A, Ferrarini M 1985 Large granular lymphocytes have a promoting activity on human peripheral blood erythroid burst-forming units. Blood 65:464-472

5. Porter PN, Ogawa M 1982 Characterization of human erythroid burst-prometing activity derived from bone marrow conditioned medium. Blood 59:1207-1212

6. Johnson GR, Metcalf $P 1977$ Pure and mixed erythroid colony formation in vito stimulated by spleen conditioned medium with no detectable erythropoietin. Proc Natl Aced Sci USA 74:3879-3882

7. Meytes D, Ma A, Ortega JA, Shore NA, Dukes PP 1979 Human erythroid burst-promoting activity produced by phytohemagglutin-stimulated raniresistant peripheral blood mononuclear cells. Blood 54:1050-1059

8. Merberg A 1980 Plasma erythropoietin levels in fetal and newborn rats: response to hypoxia. Exp Hematol 8:615-619

9. Brown MS, Garcia JF, Phibbs RH, Dallman PR 1983 Postnatal changes in erythropoietin levels in untransfused premature infants. J Pediatr 103:612617
10. Sanengen T, Halvorsen S 1985 Regulation of erythropoiesis during rapid growth. Br J Hematol 61:273-279

11. Dukes PP, Ma A, Clemons CK, Meytes D 1985 Measurement of human erythroid burst-promoting activity by a specific cell culture assay. Exp Hematol 13:59-66

12. Messier HA, Till JE, McCulloch FA 1973 Interacting cell populations affecting granulopoietic colony formation by normal and leukemic human marrow cells. Blood 42:701-710

13. Porter PN, Ogawa M, Leary AG 1980 Enhancement of the growth of human early erythroid progenitors by bone marrow conditioned media. Exp Hematoll 8:83-91

14. Wisen C, Moper Y, Speck B, Bendy J 1983 Haematopoietic stimulator and inhibitors in aplastic anaemia serum. Br J Haematol 54:519-530.

15. Zuckerman KS, Hawk M 1983 Mitogen induced stimulation and suppression of erythroid burst promoting activity production by human mononuclear cells. Br J Haematol 55:145-153

16. Nagaoki T, Moriya N, Miyawaki T, Ski H, Kubo M, Okuda T, Taniguchi N 1981 Suppression of $\mathrm{B}$ cell differentiation by dialyzable humoral factors derived from pokeweed mitogen stimulated cord $T$ cells. J Immunol 124:1563-1568

17. Arson EB, Epstein MB, Seeger RC 1979 Monocyte subsets in neonates and children. Pediatrics 64:740-744 